\title{
Conhecimento de estudantes universitários sobre a cirurgia bariátrica $(\mathrm{CB})^{*}$
}

\author{
Knowledge of students about bariatric \\ surgery (SB)
}

Fernanda Souza Lopes ${ }^{1}$ Linconl Agudo Oliveira Benito ${ }^{2}$
* Recebido em: 19/06/2016. Aprovado em: 04/10/2017.

O presente artigo surgiu do relatório final apresentado juntamente ao $14^{\circ}$ Programa de Iniciação Científica (2015-2016) do Centro Universitário de Brasília (UniCEUB).

1 Bolsista de Iniciação Científica como o projeto possuidor do título "Conhecimento de estudantes universitários sobre a cirurgia bariátrica (CB) aprovado no $14^{\circ}$ Programa de Iniciação Cientifica (2015-2016) do Centro Universitário de Brasília (UniCEUB). Graduanda em Enfermagem do Centro Universitário de Brasília (UniCEUB).

2 Orientador Projeto de Iniciação Científica. Docente do UniCEUB.

\section{Resumo}

Trata-se de um estudo transversal, descritivo e de abordagem quantitativa que analisou o conhecimento de estudantes de uma instituição de ensino superior (IES) com sede em Brasília (D.F.) sobre a CB. Foram entrevistados quatrocentos (400) estudantes. $48 \%(\mathrm{n}=192)$ cursavam "enfermagem", 24\% (n=96) cursavam "nutrição", 15,50\% (n=62) cursavam "medicina", 8,25\% (n=33) cursavam "biomedicina" e 4,25\% $(\mathrm{n}=17)$ cursavam "biologia". O perfil dos atores sociais participantes do presente estudo se constituiu de $78,75 \%(\mathrm{n}=315)$ pessoas do sexo feminino, $87,75 \%(\mathrm{n}=351)$ solteiras, $70,75 \%(\mathrm{n}=283)$ não desenvolvem atividade remunerada, 93,25\% ( $\mathrm{n}=373)$ negam enfermidade(s), 94,50\% ( $\mathrm{n}=378)$ negam tabagismo, 78\% $(\mathrm{n}=312)$ negam consumo medicamentoso, $93 \%$ $(\mathrm{n}=372)$ negam etilismo, 99,25\% ( $\mathrm{n}=397)$ negam hipertensão arterial (HA), 100\% ( $\mathrm{n}=400)$ negam diabetes mellitus (DM), 81,50\% $(\mathrm{n}=326)$ negam sobrepeso e $95 \%(n=380)$ negam obesidade. Foi identificado, ainda, reduzido conhecimento dentre os entrevistados, apenas na categoria analítica relacionada à necessidade de suplementação alimentar utilizando polivitamínicos após a realização da CB.

Palavras-chave: Conhecimento. Estudantes. Obesidade. Obesidade mórbida. Cirurgia bariátrica.

\begin{abstract}
This is a cross-sectional study, descriptive, and a quantitative approach that examined the knowledge of students from a higher education institution (HEI) with headquarters in Brasilia (DF) about the Bariatric Surgery (BS). There were four hundred respondents (400) students, and $48 \%(\mathrm{n}=192)$ were enrolled in "nursing", $24 \%(\mathrm{n}=96)$ were enrolled in "nutrition", 15.50\% ( $\mathrm{n}=62$ ) were enrolled in "medicine", 8.25\% $(\mathrm{n}=33)$ were enrolled in "biomedicine" and $4.25 \%(\mathrm{n}=17)$ were enrolled in "biology". The profile of the social actors in the present study were $78.75 \%$ $(\mathrm{n}=315)$, females, $87.75 \%(\mathrm{n}=351)$ single, $70.75 \%(\mathrm{n}=283)$ do not develop remunerated activity, 93.25\% $(n=373)$ denied disease, $94.50 \%(n=378)$ deny smoking, 78\% $(\mathrm{n}=312)$ deny drug consumption, 93\% $(\mathrm{n}=372)$ deny alcohol consumption, $9925 \%(n=397)$ deny arterial hypertension (AH), 100\% $(n=400)$ deny diabetes mellitus (DM), 81.50\% $(n=326)$ deny overweight and $95 \%(n=380)$ deny obesity. It has been identified little knowledge among the respondents, only in the analytic category related to the need for food supplementation using multivitamins after completion of the BS.
\end{abstract}

Keywords: Knowledge. Students. Obesity. Morbid obesity. Bariatric surgery. 


\section{Introdução}

A obesidade (CID10-E66) é uma condição caracterizada por anormal ou excessivo acúmulo de gordura em extensão, com vários prejuízos para a saúde. Se constitui enquanto enfermidade crônica, de complexo controle, relacionada a complicações e insucessos terapêuticos, afetando nações desenvolvidas e em desenvolvimento, caracterizada enquanto epidemia mundial e que atinge pessoas em todos os níveis socioeconômicos (OMS, 2009; TEICHMANN et al., 2006; OMS, 2000).

Ela acarreta prejuízos à saúde, como alterações na função respiratória, distúrbios dermatológicos e do aparelho locomotor, hipertensão arterial sistêmica (HAS), intolerância à glicose, hipertrofia ventricular esquerda (HVE), hiperuricemia, elevação do fibrinogênio, síndrome metabólica (SM), síndrome da apneia obstrutiva do sono (SAOS), dislipidemias, doenças cardiovasculares, diabetes mellitus tipo 2 (DM2) e certos tipos de câncer (CA) (FELIX; SOARES; NÓBREGA, 2012; TAVARES et al., 2012; COUTINHO, 2007; PINHEIRO; FREITAS; CORSO, 2004).

Nos Estados Unidos (EUA), há mais de 15,0 milhões de pessoas obesas graves e no Brasil, mais de 38,8 milhões de pessoas com 20 anos ou mais, se encontram acima do peso. Destes, $41,1 \%$ são pessoas do sexo masculino, que se encontraram acima do peso, e 8,9\% se apresentam obesas. Quanto ao sexo feminino, $40 \%$ estavam com sobrepeso e 13,1\% com obesidade (TAVARES et al., 2012; STURM, 2007; IBGE, 2004; OMS, 2000).

Opções clássicas do tipo não cirúrgicas incluem a restrição dietética, a atividade física, as modificações comportamentais, o uso de medicamentos e o suporte psicológico, cujos resultados, frequentemente são limitados e obtidos em curtos períodos de tempo (TAVARES et al., 2012; COUTINHO, 2007; SCHNEIDER; MUN, 2005).

Os insucessos dos tratamentos clínicos para a obesidade e obesidade mórbida são superiores a 90\% e, dessa forma, alternativas terapêuticas têm sido propostas, como o tratamento cirúrgico, sendo apontado enquanto promissor para perda de peso significativa e duradoura. A CB como opção de tratamento, é indicada para pacientes com índice de massa corporal (IMC) a partir de $40 \mathrm{~kg} /$ $\mathrm{m}^{2}$ (obesidade grave) ou acima de $35 \mathrm{~kg} / \mathrm{m}^{2}$ na presença de morbidades associadas (FELIX; SOARES; NÓBREGA, 2012; SANTOS et al., 2012; VALEZI et al., 2008). procedimento invasivo que pode causar algumas complicações advindas de sua implementação. O procedimento mais realizado tem sido a Derivação Gástrica em Y de Roux (DGYR) (ROCHA, 2012; SBCBM, 2006; LÓPEZ-FONTANA; FONERÓN; CASTILLO, 2012).

Nesse sentido e, segundo o Ministério da Saúde (MS) e a Sociedade Brasileira de Cirurgia Bariátrica e Metabólica (SBCBM), a CB vem sendo realizada em grande escala, tanto pelos serviços públicos quanto pela iniciativa privada, conforme a tabela abaixo:

Tabela 01 - Frequência de cirurgias bariátricas realizadas no Brasil, entre os anos de 2003 a 2012, por fonte financiadora ( $n=437.638)$ :

\begin{tabular}{c|c|c|c}
\hline Ano & $\begin{array}{c}\text { Rede particular, } \\
\text { suplementar e SUS (\%) }\end{array}$ & $\begin{array}{c}\text { Rede pública (SUS) } \\
(\%)\end{array}$ & Total $(\%)$ \\
\hline 2012 & $72.000(17,85)^{* * * *}$ & $6.029(17,65)$ & $78.029(17,85)$ \\
\hline 2011 & $70.000(17,35)^{* * *}$ & $5.332(15,6)$ & $75.332(17,2)$ \\
\hline 2010 & $60.000(14,9)^{* *}$ & $4.489(13,15)$ & $64.489(14,75)$ \\
\hline 2009 & $45.000(11,15)^{*}$ & $3.731(10,95)$ & $48.731(11,15)$ \\
\hline 2008 & $38.000(9,4)$ & $3.139(9,2)$ & $41.139(9,4)$ \\
\hline 2007 & $33.000(8,2)$ & $2.974(8,7)$ & $35.974(8,2)$ \\
\hline 2006 & $29.500(7,3)$ & $2.528(7,4)$ & $32.028(7,3)$ \\
\hline 2005 & $22.000(5,45)$ & $2.266(6,65)$ & $24.266(5,55)$ \\
\hline 2004 & $18.000(4,45)$ & $1.872(5,5)$ & $19.872(4,55)$ \\
\hline 2003 & $16.000(3,95)$ & $1.778(5,2)$ & $17.778(4,05)$ \\
\hline Total & $403.500(100)$ & $34.138(100)$ & $437.638(100)$ \\
\hline
\end{tabular}

Fonte: Ministério da Saúde (MS). Sociedade Brasileira de Cirurgia Bariátrica e Metabólica (SBCBM), 2015.

* Destas 25\%foram realizadas por videolaparotomia.

** Destas 35\% foram realizadas por videolaparotomia.

*** Destas $40 \%$ foram realizadas por videolaparotomia.

**** Destas 75\% foram realizadas por videolaparotomia.

Um outro tipo de CB é o de gastroplastia a Fobi-Capella. A taxa de mortalidade desta pode chegar até $1 \%$ nas grandes séries e as complicações gerais podem chegar até $40 \%$. Dentre as complicações verificadas, podem ser citadas dificuldades na realização de procedimentos de intubação, o surgimento de fístula da anastomose gastrojejunal, hematoma-seroma da incisão, hérnia incisional, embolia pulmonar, atelectasia e pneumonia pós-operatória, náuseas e vômitos persistentes, estenose da anastomose gastrojejunal, complicações com o anel, obstrução intestinal, síndrome de dumping, colecistite calculosa e complicações metabólicas como a deficiência de vitamina $\mathrm{B}_{12 \mathrm{e}}$ ferro (Fe) (GEMPELERA; DÍAZB; SARMIENTO, 2012; SCHAUER et al., 2012; YESTE; CARRASCOSA, 2012; ILIAS, 2011; ASTUDILLO; NEIRA, 2010; RAVELLI et al., 2007).

As cirurgias que apresentam melhores resultados podem reduzir o peso entre 30 e $40 \%$ e esse efeito pode ser mantido por longos períodos. Outra questão relacio- 
nada a esse procedimento se refere ao aumento do peso, que ocorre mais frequentemente entre 03 e 06 anos depois da cirurgia. Em outros estudos, se tem considerado que o reganho de peso tem favorecido o reaparecimento de comorbidades com o DM (MANIGLIA et al., 2012; MELO; SÃO-PEDRO, 2012; PAPAPIETRO, 2012; PAZZIANOTTO-FORTI et al., 2012; PAREJA; PILLA; GELONEZE, 2006).

Fenômenos de fundo psíquico e emocional, também, são referidos na literatura científica em decorrência do desenvolvimento desse procedimento, como casos onde um incremento da agressividade é referido pelos pacientes após sua realização, suicídio de pacientes previamente depressivos após a CB em uma amostra de 16.683 pacientes operados na Pensilvânia, surgindo a necessidade de estudos sobre o impacto da $\mathrm{CB}$ sobre as comorbidades psiquiátricas, principalmente em relação ao autoextermínio (ADAMS et al., 2007; LEAL; BALDIN, 2007; OMALU et al., 2005).

Nesse sentido, se constituíram enquanto objetivos do presente estudo, analisar o conhecimento de estudantes universitários de uma instituição de ensino superior (IES), com sede na cidade de Brasília, Distrito Federal (D.F.) sobre a cirurgia bariátrica (CB) e construir o perfil sociodemográfico de estudantes universitários atores sociais participantes da presente pesquisa.

\section{Metodologia}

Trata-se de um estudo transversal, descritivo e potencializado por uma dimensão quantitativa. Para aquisição de subsídios necessários à edificação do presente estudo, foi utilizado um instrumento de coleta de dados (ICD) proposto por Garrido Jr (2004), possuidor do título "Teste de entendimento aos pacientes candidatos à cirurgia da obesidade mórbida (TEPCCOM)”, sendo viável dessa forma a aquisição das fontes primárias.

As fontes secundárias derivaram de artigos de periódicos científicos, produções acadêmicas e legislação correlata, adquirida após buscas bibliográficas eletrônicas junto à base de dados informatizados nacionais e internacionais (Biblioteca Virtual em Saúde - BVS, Cuiden, Embase, Minerva-UFRJ, Pubmed, Saber-USP).

Foram utilizados os Descritores em Ciências da Saúde (DeCS) da Biblioteca Virtual da Saúde (BVS) sendo estes "Conhecimento" com o número de registro “33182” e identificador único “D019359”, "Estudantes” com o número de registro "13721" e identificador único "D013334", "Peso Corporal” com o número de registro "1860" e identificador único "D001835", "Obesidade” com o número de registro "9951" e identificador único “D009765”, “Obesidade Mórbida” com o número de registro “19272” e identificador único "D009767”, “Cirurgia Bariátrica” com o número de registro “51221” e identificador único "D050110”.

Nas combinações e conjugação dos descritores em ciências da saúde selecionados, foram utilizados os operadores lógicos booleanos de pesquisa "and", "or" e "and not", permitindo desta forma potencializar o processo de identificação e aquisição das referências. Foram selecionadas referencias nos idiomas "português", "espanhol" e "inglês".

Objetivando respeitar, integralmente, os dispositivos expostos juntamente à resolução de número 466/2012 do Conselho Nacional de Saúde (CNS) do Ministério da Saúde (MS), relacionada às "diretrizes e normas regulamentadoras em pesquisa utilizando seres humanos", um projeto de pesquisa foi submetido à apreciação e avaliação bioética junto ao Comitê de Ética e Pesquisa (CEP) do Centro Universitário de Brasília (UniCEUB), sendo o mesmo aprovado com o número da CAAE “50679015.6.0000.0023”.

O termo de conhecimento livre e esclarecido (TCLE) foi lido e explicado para todos os participantes da presente pesquisa que, após concordarem com o seu teor, assinaram o mesmo. As entrevistas ocorreram entre os meses de fevereiro e abril do ano de 2016, respeitando a disponibilidade dos depoentes.

Se constituíram enquanto critérios de inclusão da presente pesquisa, estudantes universitários da área da saúde, tanto do sexo feminino quanto do sexo masculino, com idade igual ou superior a dezoito (18) anos, que estivessem regularmente inscritos junto a instituição de ensino superior (IES) participante da pesquisa, que estivessem regularmente inscrito junto ao primeiro período $\left(1^{\circ}\right)$ do ano de 2016, que estivessem regularmente inscritas junto ao período matutino e no noturno do ano de 2016, que possuíssem disponibilidade e quisessem participar livremente da presente pesquisa.

Após a aquisição dos dados, estes foram organizados, sendo construído um banco de dados, utilizando para tal atividade o software Microsoft Excel $2013^{\circledR}$, pertencente ao pacote Microsoft Office $2013^{\circledR}$, for Windows ${ }^{\circledast}$. Os resultados foram expostos por meio de tabelas explicativas. 


\section{Resultados}

Por meio do presente estudo, foi permitido verificar que o perfil socioeconômico e demográfico dos graduandos em saúde participantes se constituiu de $48 \%$ $(\mathrm{n}=192)$ dos entrevistados declararam cursar enfermagem, $78,75 \%(n=315)$ eram do sexo feminino, $87,75 \%$ $(n=351)$ se encontravam solteiras, $70,75 \%(n=283)$ não desenvolvem atividade remunerada, 93,25\% ( $\mathrm{n}=373)$ negam enfermidade(s), 94,50\% ( $n=378)$ negam tabagismo, $78 \%(\mathrm{n}=312)$ negam consumo medicamentoso, 93\% ( $\mathrm{n}=372)$ negam etilismo, 99,25\% ( $\mathrm{n}=397)$ negam hipertensão arterial (HA), 100\% $(\mathrm{n}=400)$ negam diabetes mellitus (DM), 81,50\% ( $\mathrm{n}=326)$ negam sobrepeso e 95\% $(\mathrm{n}=380)$ negam obesidade, conforme exposto junto a tabela de número 02 .

Tabela 02 - Perfil socioeconômico e demográfico dos atores sociais participantes do presente estudo, por categorias analíticas, Brasília, Distrito Federal (D.F.), Brasil, $(n=400)$.

\begin{tabular}{|c|c|c|c|c|c|c|c|}
\hline \multirow{2}{*}{ Categorias analíticas } & & \multicolumn{2}{|c|}{ Total } & \multicolumn{2}{|c|}{ Feminino } & \multicolumn{2}{|c|}{ Masculino } \\
\hline & & $n$ & $\%$ & $n$ & $\%$ & $n$ & $\%$ \\
\hline \multicolumn{8}{|l|}{ Curso } \\
\hline & Enfermagem & 192 & 48 & 167 & 53 & 25 & 29,4 \\
\hline & Nutrição & 96 & 24 & 86 & 27,3 & 10 & 11,75 \\
\hline & Medicina & 62 & 15,5 & 30 & 9,5 & 32 & 37,65 \\
\hline & Biomedicina & 33 & 8,25 & 22 & 7 & 11 & 12,95 \\
\hline & Biologia & 17 & 4,25 & 10 & 3,2 & 7 & 8,25 \\
\hline \multicolumn{8}{|l|}{ Estado civil } \\
\hline & Solteira & 351 & 87,75 & 272 & 86,35 & 79 & 92,95 \\
\hline & Casado & 39 & 9,75 & 33 & 10,45 & 6 & 7,05 \\
\hline & União estável & 6 & 1,5 & 6 & 1,9 & - & - \\
\hline & Divorciada & 2 & 0,5 & 2 & 0,65 & - & - \\
\hline & Separada & 2 & 0,5 & 2 & 0,65 & - & - \\
\hline \multicolumn{8}{|l|}{ Atividade remunerada } \\
\hline & Não & 283 & 70,75 & 220 & 69,85 & 61 & 71,75 \\
\hline & $\operatorname{sim}$ & 116 & 29,0 & 94 & 29,85 & 24 & 28,25 \\
\hline & Não informado & 1 & 0,25 & 1 & 0,3 & - & - \\
\hline \multicolumn{8}{|l|}{ Possui enfermidade(s) } \\
\hline & Não & 373 & 93,25 & 290 & 92,05 & 82 & 96,5 \\
\hline & Sim & 27 & 6,75 & 25 & 7,95 & 3 & 3,5 \\
\hline \multicolumn{8}{|l|}{ Tabagista } \\
\hline & Não & 378 & 94,5 & 305 & 96,85 & 75 & 88,25 \\
\hline & Sim & 22 & 5,5 & 10 & 3,15 & 10 & 11,75 \\
\hline \multicolumn{8}{|l|}{ Consume medicamentos } \\
\hline & Não & 312 & 78 & 232 & 73,65 & 80 & 94,1 \\
\hline & Sim & 88 & 22 & 83 & 26,35 & 5 & 5,9 \\
\hline \multicolumn{8}{|l|}{ Etilista } \\
\hline & Não & 372 & 93 & 300 & 95,25 & 72 & 84,7 \\
\hline & Sim & 28 & 7 & 15 & 4,75 & 13 & 15,3 \\
\hline \multicolumn{8}{|l|}{ Hipertensão arterial } \\
\hline & Não & 397 & 99,25 & 315 & 100 & 83 & 97,65 \\
\hline & Sim & 3 & 0,75 & - & - & 2 & 2,35 \\
\hline \multicolumn{8}{|l|}{ Diabetes mellitus } \\
\hline & Não & 400 & 100 & 315 & 100 & 85 & 100 \\
\hline \multicolumn{8}{|l|}{ Sobrepeso } \\
\hline & Não & 326 & 81,5 & 258 & 81,90 & 68 & 80 \\
\hline & Sim & 74 & 18,5 & 57 & 18,10 & 17 & 20 \\
\hline \multicolumn{8}{|l|}{ Obesidade } \\
\hline & Não & 380 & 95 & 300 & 95,25 & 80 & 94,1 \\
\hline & Sim & 20 & 5 & 15 & 4,75 & 5 & 5,9 \\
\hline Total & & 400 & 100 & 315 & 100 & 85 & 100 \\
\hline
\end{tabular}

Fonte: Produção dos autores, 2016.

Já em relação às repostas disponibilizadas no que se refere aos questionamentos desenvolvidos, foi identificado reduzido conhecimento dentre os entrevistados, apenas na categoria analítica relacionada a necessidade de suplementação alimentar utilizando polivitamínicos após a realização da $\mathrm{CB}$, conforme exposto junto a tabela de número 03 . 
Tabela 03 - Respostas aos questionamentos desenvolvidos, por frequência e percentual, Brasília, Distrito Federal (D.F.), Brasil, ( $n=400)$ :

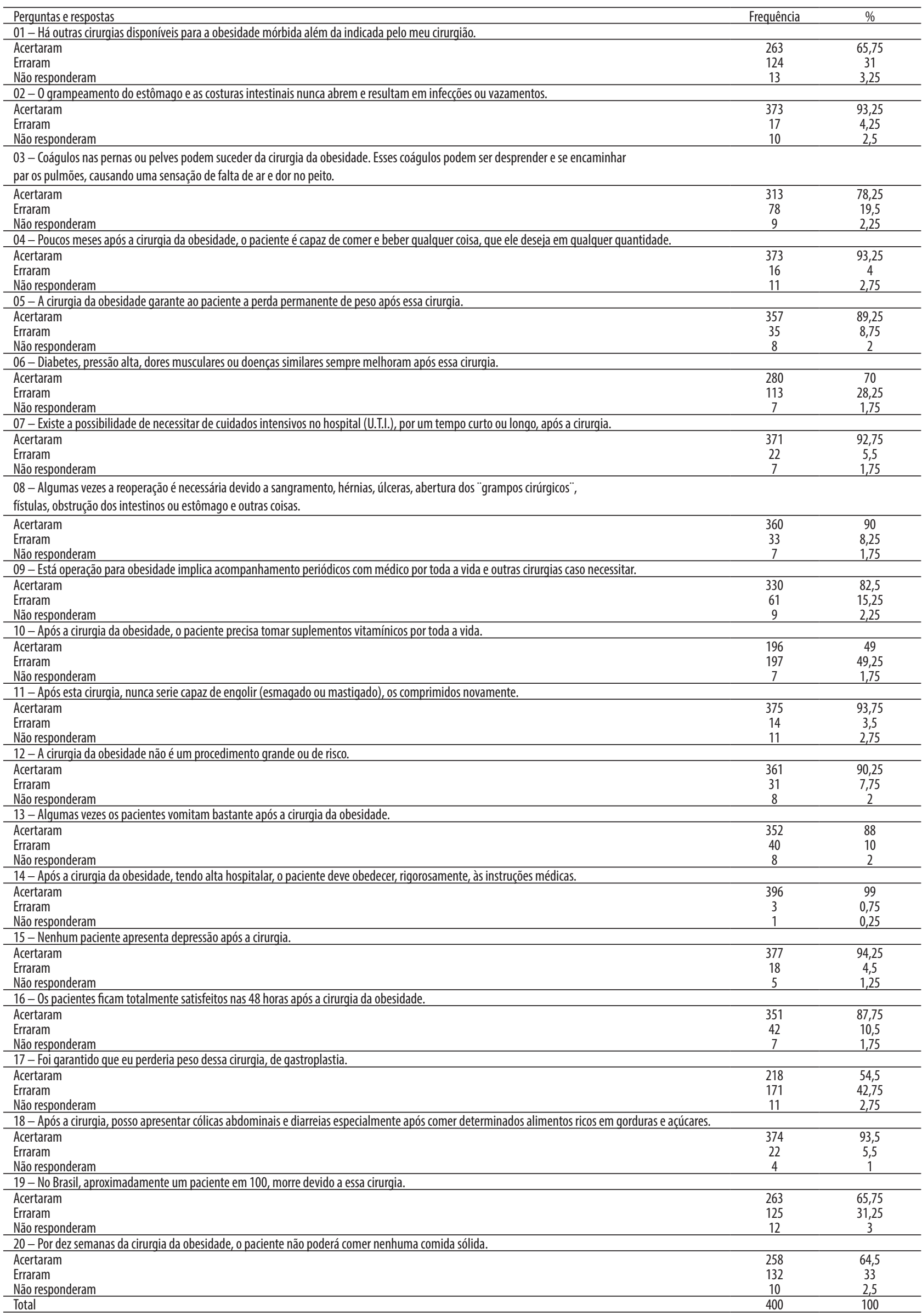


As deficiências nutricionais, após realização da $\mathrm{CB}$, têm sido cada vez mais relatadas e analisadas juntamente à literatura científica, sendo muito frequentes, em razão da redução na ingestão oral e na absorção de nutrientes (MUSTAFA, 2014; TOREZAN, 2013; QUEIROZ; HAACK; MUNIZ, 2012; BORDALO; MOURÃO; BRESSAN, 2011; MECHANICK et al., 2008; KUSHNER, 2006; MALINOWSKI, 2006; BLOOMBERG et al., 2005; DEITEL; COWAN, 2000).

Nesse sentido, todos os pacientes submetidos ao procedimento operatório bariátrico, devem receber suplementação de vitaminas e minerais diariamente. Entretanto, ainda não existe consenso em relação a melhor dose de suplementação dos micronutrientes a serem consumidos pela pessoa cirurgiada (TOREZAN, 2013; MALINOWSKI, 2006).

Numa recente publicação, é apontado que as quantidades iniciais de suplementação de vitaminas e micronutrientes para normalizar os marcadores biológicos, dois (2) anos após a realização da CB para pelo menos 3000 mg de cálcio (Ca) com 7000 UI de vitamina D, 50.000 UI de vitamina A, $40 \mathrm{mg}$ de zinco (Z), e de 200 $\mathrm{mg}$ de ferro $(\mathrm{Fe})$, devem ser prescritos inicialmente (TOPARD et al., 2014).

Após a realização da $\mathrm{CB}$, há a necessidade do uso de suplementação vitamínica diária por toda vida do paciente, sendo recomendados um (01) a dois (02) comprimidos de suplementos polivitamínico, por via oral e diariamente (RAMOS; MELLO, 2015; QUEIROZ; HAACK; MUNIZ, 2012; BORDALO; MOURÃO; BRESSAN, 2011).

Alguns autores já sugerem doses adicionais além das doses presentes nos polivitamínicos usados de rotina, sendo o caso da vitamina $\mathrm{B} 12, \mathrm{Fe}, \mathrm{Z}, \mathrm{Ca}$, da vitamina D, da vitamina A e da vitamina E. Entretanto, a adesão a essa recomendação é variável, devido a fatores, como por exemplo, o não reconhecimento da importância do uso destes, fatores econômicos e a ainda, a presença de múltiplas informações, algumas vezes conflituosas, a respeito da formula ideal de reposição (RAMOS; MELLO, 2015; TOREZAN, 2013; MALINOWSKI, 2006).

Estudos mostram que essas deficiências nutricionais são frequentes em pessoas que se encontrem gravemente obesas ou obesas mórbidas, mesmo antes do procedimento cirúrgico, e que a $\mathrm{CB}$ pode agravar o quadro. As deficiências nutricionais no pós-operatório (PO) podem ser atribuídas a diversos fatores como a deficiência pré-cirúrgica, a ingestão alimentar reduzida, a suplementação inadequada, a má-absorção de nutrientes e a terapia nutrológica inadequada, como a reduzida monitorização ou ainda, segundo alguns autores, pela ausência de seguimento no PO (RAMOS; MELLO, 2015; RAVELLI et al., 2007; YOSSEF et al., 2007; CARLIN et al., 2006; BLOOMBERG et al., 2005).

Cada técnica da CB, promove alterações digestivas e absortivas características, necessitando de um programa educativo multidisciplinar exclusivo e direcionado tanto no pré quanto no pós-operatório, enfatizando os hábitos de atividade física e a necessidade de aderir às restritas recomendações dietéticas (RAVELLI et al., 2007; BLOOMBERG et al., 2005; ALVAREZ-LEITE, 2004).

Nas pessoas submetidas à $\mathrm{CB}$, principalmente pelas técnicas de by-pass gástrico em Y-de-Roux (BGYR) ou a derivação biopancreática/Duodenal Switch (DBP/ DS), estas apresentam um maior risco em desenvolver deficiências nutricionais, devido, principalmente, à exclusão de partes do trato gastrointestinal. Esses fenômenos prejudicam a absorção eficiente dos nutrientes, evidenciando que as técnicas cirúrgicas com características disabsortivas exercem um maior impacto na absorção de vitaminas e minerais e frequentemente, resultam em deficiências nutricionais (RAMOS; MELLO, 2015; BORDALO; MOURÃO; BRESSAN, 2011).

As técnicas de $\mathrm{CB}$ do tipo disabsortivas são as que promovem maior incidência de deficiência dos micronutrientes, que é proporcional ao comprimento da área desviada e à porcentagem de perda de peso. As cirurgias mistas com maior componente disabsortivo acarretam mais carências nutricionais do que aquelas com menor disabsorção (MUSTAFA, 2014; QUEIROZ; HAACK; MUNIZ, 2012; BLOOMBERG et al., 2005; ALVAREZ-LEITE, 2004)

Numa pesquisa desenvolvida na modalidade de estudo de caso clínico, realizado com uma paciente com 25 anos de idade e com diagnóstico de obesidade grau III que engravidou após três (03) meses de cirurgia, esta aponta para deficiências de alguns nutrientes como ferro $(\mathrm{Fe})$, vitaminas do complexo B (B1, B2, B3, B5, B6, B7, B9, B12), vitaminas lipossolúveis, sendo necessária prescrição de suplementação objetivando reverte o quadro em questão (MUSTAFA, 2014; QUEIROZ; HAACK; MUNIZ, 2012; MATTOS et al., 2010).

Dessa forma, após a realização da $\mathrm{CB}$, os hábitos alimentares do paciente sofrem uma série de mudanças 
significativas, surgindo, dessa forma, a necessidade do acompanhamento com uma equipe multiprofissional de saúde, fornecendo condições para que seja percebida a amplitude do processo ao qual este foi submetido para tratamento da obesidade ou obesidade mórbida (RAMOS; MELLO, 2015; TOREZAN, 2013; MATTOS et al., 2010; RAVELLI et al., 2007).

Após a realização da $\mathrm{CB}$ em suas várias modalidades, várias são as substâncias que apresentam carência juntamente ao organismo, sendo principalmente as vitaminas hidrossolúveis (vitamina B12, ácido fólico, vitamina $B 1$ ), as vitaminas lipossolúveis (vitamina $D$, vitamina $A$, vitamina $\mathrm{E}$, vitamina $\mathrm{K}$ ), além da deficiência de elementos traço (ferro, cobre, zinco e selênio) (TOREZAN, 2013; QUEIROZ; HAACK; MUNIZ, 2012).

Já em outros estudos, a CB está associada a alterações no metabolismo ósseo, perda óssea e a um possível aumento de fraturas, sendo necessário realizar juntamente a esse paciente, uma intervenção nutricional aliada à suplementação regular de vitamina $\mathrm{D}$ e cálcio (Ca), além de uma vida saudável (RAMOS; MELLO, 2015; MUSTAFA, 2014; TOREZAN, 2013; MALINOWSKI, 2006).

As referidas deficiências nutricionais associadas às $\mathrm{CB}$ são percebidas devido aos desvios desenvolvidos juntamente as estruturas intestinais, nos quais há inúmeros sítios absortivos de nutrientes. A CB promove, ainda, uma redução severa no consumo alimentar, o que acarreta a ingestão de dietas do tipo hipocalóricas e deficientes em micronutrientes, permitindo consequentes complicações nutricionais (MUSTAFA, 2014; TOREZAN, 2013; RAVELLI et al., 2007; KUSHNER, 2006; BLOOMBERG et al., 2005; DEITEL; COWAN, 2000).

Devido à baixa ingestão alimentar por esse paciente, este possui a necessidade de suplementação de vitaminas e de minerais o mais breve possível. Nesse sentido, a suplementação nutricional, geralmente, não se inicia logo após a $\mathrm{CB}$, devido às dificuldades verificadas em ingerir comprimidos e, também, às intolerâncias causadas por eles (TOREZAN, 2013; RAVELLI et al., 2007; CRUZ; MORIMOTO, 2004; GARRIDO JR et al., 2002; MACLEAN; RHODE; SHIZGAL, 1983).

Objetivando prevenir o surgimento de complicações no PO de CB, alguns apontamentos são propostos pela literatura científica corrente. $\mathrm{O}$ sucesso da $\mathrm{CB}$ a longo prazo passa pelo seguimento das orientações dietéticas, ou seja, aumentar a consistência dos alimentos gradualmente, ao longo de um período de 1 a 2 meses, avançando de líquidos para uma dieta leve e, finalmente, uma dieta regular com o objetivo de evitar deficiências nutricionais (FAÉ; LIBERALI; COUTINHO, 2015; RAMOS; MELLO, 2015; LO MENZO, 2014; TOREZAN, 2013; BORDALO; MOURÃO; BRESSAN, 2011).

$\mathrm{O}$ tratamento das deficiências nutricionais desses pacientes deve ser composto de "megadoses" de micronutrientes, se levando em consideração a reduzida biodisponibilidade, a menor ingestão e a área de absorção, decorrente do ato cirúrgico operatório desenvolvido (FAÉ; LIBERALI; COUTINHO, 2015; TOREZAN, 2013).

Nesse sentido, é de fundamental importância a prescrição de multivitaminas e suplementação mineral, para tratamento da anemia no $\mathrm{PO}$ da $\mathrm{CB}$. Já a realização de triagem de deficiências por meio de exames laboratoriais, individualizando algumas reposições, permite uma melhor adequação de suplementação ou do tratamento de deficiências especificas (FAÉ; LIBERALI; COUTINHO, 2015; MUSTAFA, 2014; RAMOS; MELLO, 2015; KWON et al., 2014).

Dessa forma, é fundamental o acompanhamento clínico nutricional de todas as pessoas submetidas à $\mathrm{CB}$, a fim de garantir a manutenção do processo de perda de peso de forma saudável e de qualidade (MUSTAFA, 2014; TOREZAN, 2013; BORDALO; MOURÃO; BRESSAN, 2011).

\section{Conclusão}

Os objetivos traçados juntamente ao presente estudo foram alcançados integralmente, além da metodologia de pesquisa utilizada permitir a realização das atividades propostas com eficiência e qualidade.

Por meio do presente estudo, foi possível verificar que os estudantes universitários entrevistados, possuem conhecimento no que se refere a $\mathrm{CB}$ em seus vários aspectos. Entretanto, especificidades relacionadas a esse procedimento cirúrgico operatório, por exemplo, em relação ao consumo de polivitaminicos após a sua realização dessa cirurgia, não se constitui enquanto questão de conhecimento pleno dos atores sociais participante da presente pesquisa.

Por esta pesquisa ter entrevistados pessoas regularmente inscritas juntamente a cursos da área da saúde, esse fator contribuiu para que estas possuíssem conhecimentos no que se refere ao procedimento cirúrgico operatório em questão. Resultados diferentes aqueles 
encontrados no presente estudo, podem ser identificados se os depoentes participantes estiverem matriculados em outras áreas e cursos representantes de outros campos do conhecimento.

Uma outra evidência verificada na presente pesquisa foi a "poderosa" e "hegemônica" influência dos meios de comunicação, em todas as suas categorias e dimensões, permitindo nesse sentido, um maior conhecimento das questões relacionadas ao procedimento cirúrgico operatório de redução do peso corporal analisado.

Essas influências podem contribuir, diretamente, para que as pessoas de uma forma geral, possuam um maior conhecimento relativo, no que se refere a produtos, procedimentos e técnicas propostas, relacionados aos novos avanços no campo científico da saúde.

Por outro lado, a necessidade de redução e controle do peso corporal elevado de pessoas que se encontram obesas ou obesas mórbidas, além do tratamento de enfermidades e comorbidades relacionadas, ampliam o quantitativo daquelas que aderem a esse procedimento cirúrgico, enquanto um dos últimos recursos para o combate de controle de doenças pré-existentes e posterior reestabelecimento à saúde.

Entretanto, esse procedimento cirúrgico delicado, e que provocará grandes modificações juntamente à estrutura corporal da pessoa a ele submetido, não pode e nem deve ser indicado a pessoas que dele não necessitem verdadeiramente ou que possuam interesses de ordem apenas estética.

Nesse sentido, é sugerido que outros estudos e pesquisas que versem sobre tão importante temática na contemporaneidade sejam implementados e incentivados, objetivando ampliar o entendimento desta, permitindo maior conhecimento e elucidação dos efeitos emanados pela sua implementação.

\section{Agradecimentos}

Agradecemos a Assessoria de Pós-Graduação e Pesquisa na pessoa da Prof. Dra. Fernanda Costa Vinhaes de Lima, pela atenção, dedicação e constante apoio na realização da presente pesquisa.

Ao Centro Universitário de Brasília (UniCEUB), pelo pioneirismo e iniciativa de disponibilização de uma bolsa de iniciação científica, sem a qual a presente pesquisa seria inviável.

\section{Referências}

bypass surgery. New England Journal of Medicine, Boston, v. 357, n. 8, p. 753-761. ago. 2007.

ALVAREZ-LEITE, J. I. Nutrient deficiencies secondary to bariatric surgery. Current Opinion in Clinical Nutrition \& Metabolic Care, London, v. 7, n. 5, p. 569-575, set. 2004 .

ASTUDILLO, J. J. A.; NEIRA, P. A. Cirugía de la obesidad en niños y adolescentes. Rev Ecu. Ped, Quito, v. 10, n. 2, p. $38-42$, Oct. 2010.

BLOOMBERG, R. D. et al. Nutritional deficiencies following bariatric surgery: what have we learned? Obes Surg, New York, v. 15, n. 2, p. 145-154, fev. 2005.

BORDALO, L. A.; MOURÃO, D. M.; BRESSAN, J. Deficiências nutricionais após cirurgia bariátrica: por que ocorrem? Acta Med Port, Lisboa, v. 24, S 4, p. 1021-1028, 2011.

CARLIN, A. M. et al. Prevalence of vitamin D depletion among morbidly obese patients seeking gastric bypass surgery. Surgery for Obesity and Related Diseases, New York, v. 2, n. 2, p. 98-103, Mar./Apr. 2006.

COSTA, D. da. Eficiência do acompanhamento nutricional no pré e pós-operatório da cirurgia bariátrica. Revista Brasileira de Obesidade, Nutrição e Emagrecimento RBONE, São Paulo, v. 7, n. 39, p. 57-68, Maio/Jun. 2013.

COUTINHO, W. Etiologia da obesidade. Rev. ABESO, São Paulo, v. 7, n. 30, p. 1-14, Dec. 2007.

CRUZ, M. R. R.; MORIMOTO, I. M. I. Intervenção nutricional no tratamento cirúrgico da obesidade mórbida: resultados de um protocolo diferenciado. Rev Nutrição, Campinas, v. 17, n. 2, p. 263-272, Apr./Jun. 2004.

DEITEL, M.; COWAN JR, G. S. M. Update: surgery for the morbidly obese patient. Canada: FD-Communications, 2000.

FAÉ, C.; LIBERALI, R.; COUTINHO, V. F. Deficiência de nutrientes a longo prazo no pós-operatório de cirurgia bariátrica: revisão sistemática. SaBios: Rev. Saúde e Biol., Campo Mourão, v. 10, n. 2, p. 46-53, maio/ago. 2015.

FELIX, L. G.; SOARES, M. J. G. O.; NÓBREGA, M. M. L. Protocolo de assistência de enfermagem ao paciente em pré e pós-operatório de cirurgia bariátrica. Rev. Bras. Enferm, Brasília, v. 65, n. 1, p. 83-91, jan./fev. 2012.

GARRIDO JR, A. B. et al. Cirurgia da obesidade. São Paulo: Atheneu: 2002.

GEMPELERA, F. E.; DÍAZB, L.; SARMIENTO, L. Manejo de la vía aérea em pacientes llevados a cirugía bariá- 
trica en el Hospital Universitario de San Ignacio, Bogotá, Colombia. Rev Colomb Anestesiol, Bogotá, v. 40, n. 2, p. 119-123, abril./jun. 2012.

ILIAS, E. J. Quais as complicações mais frequentes da gastroplastia a Fobi-Capella? Como tratá-las? Rev Assoc Med Bras, São Paulo, v. 57, n. 4, p. 365-366, jul./ago. 2011.

INSTITUTO BRASILEIRO DE GEOGRAFIA E ESTATÍSTICA. Diretoria de Pesquisas. Coordenação de Índices de Preços. Pesquisa de Orçamentos Familiares 2002-2003. Rio de Janeiro: IBGE, 2004.

KUSHNER, R. F. Micronutrient deficiencies and bariatric surgery. Curr Opin Endocinol Diab. Obes., London, v. 13, n. 5, p. 405-411, Oct., 2006. doi: 10.1097/01. med.0000244220.53163.85

KWON, Y. et al. Anemia, iron and vitamin B 12 deficiencies after Sleeve gastrectomy compared to Roux-en-Y gastric bypass: a meta-analysis. Surgery for Obesity and Related Diseases, New York, v. 10, n. 4, p. 589-597, Jul./ Aug. 2014.

LEAL, C. W.; BALDIN, N. O impacto emocional da cirurgia bariátrica em pacientes com obesidade mórbida. Rev Psiquiatr, Porto Alegre, v. 29, n. 3, p. 324-327, set./ dez. 2007.

LO MENZO, E. et al. Nutritional implications of obesity: before and after bariatric surgery. Bariatric Surgical Practice and Patient Care, New Rochelle, v. 9, n. 1, p. 9-17, mar. 2014.

LOPEZ-FONTANA, G.; FONERON V, A.; CASTILLO C, O. A. Nefrolitotomía percutánea en pacientes con cirugía bariátrica: ¿mayor riesgo de complicación?. Rev Chil Cir, Santiago, v. 64, n. 5, p. 468-471, oct. 2012.

MACLEAN, L. D.; RHODE, B. M.; SHIZGAL, H. M. Nutrition following gastric operations for morbid obesity. Ann Surg, Philadelphia, v. 198, n. 3, p. 347-355, set. 1983.

MALINOWSKI, S. S. Nutritional and metabolic complications of bariatric surgery. Am J Med Sci., New York, v. 331, n. 4, p. 219-225, Apr. 2006.

MANIGLIA, F. P. et al. Alterações e adaptações hormonais relacionadas ao apetite no pós-operatório de cirurgia bariátrica. Rev Bras Nutr Clin., Porto Alegre, v. 27, n. 3, p. 199-203, jul./set. 2012.

MATTOS, F. C. C. et al. Adesão e eficácia do acompanhamento nutricional no pré-operatório de pacientes submetidos a gastroplastia redutora em Y de Roux em um Hospital Federal Militar do Rio de Janeiro. Mn - Metabólica. São Paulo, v. 11, n. 1, p. 05-11, jan./mar. 2010.

MECHANICK, J. et al. Clinical Practice Guidelines for the Perioperative Nutritional, Metabolic, and Nonsurgical Support of the Bariatric Surgery Patient-2013 Update: Cosponsored by American Association of Clinical Endocrinologists, The Obesity Society, and American Society for Metabolic \& Bariatric Surgery. Surgery for Obesity and Related Diseases, New York, v. 4, n. 1, p. 109-184, mar. 2008.

MELO, I. T.; SÃO-PEDRO, M. Dor musculoesquelética em membros inferiores de pacientes obesos antes e depois da cirurgia bariátrica. ABCD Arq Bras Cir Dig., São Paulo, v. 25, n. 1, p. 29-32, jan./mar., 2012.

MUSTAFA, S. A cirurgia bariátrica e a perda óssea. International Journal of Nutrology, Catanduva, v. 7, n. 1, p. 22-30, jan./abr., 2014.

OMALU, B. I. et al. Suicides following bariatric surgery for the treatment of obesity. Surg Obes Relat Dis., New York, v. 1, n. 4, p. 447-449, Jul./Aug. 2005.

PAPAPIETRO, V. K. Reganancia de peso después de la cirugía bariátrica. Rev. Chilena de Cirugía, Santiago, v. 64, n. 1, p. 83-87, fev. 2012

PAREJA, J. C.; PILLA, V. F.; GELONEZE, B. N.; Operational mechanisms of anti-obesity surgeries. Einstein, São Paulo, v. 4, supl.1, p. S120-S124, 2006.

PAZZIANOTTO-FORTI, E. M. et al. Aplicação da pressão positiva contínua nas vias aéreas em pacientes em pós-operatório de cirurgia bariátrica. Fisioter Pesq., São Paulo, v. 19, n. 1, p. 14-19, jan./mar. 2012.

PINHEIRO, A. R. O.; FREITAS, S. F. T.; CORSO, A. C. T. Uma abordagem epidemiológica da obesidade. Rev Nutr., Campinas, v. 17, n. 4, p. 523-533, dez. 2004.

QUEIROZ, C. S.; HAACK, A.; MUNIZ, L. B. Caso clínico de gestante com obesidade grau III submetida à cirurgia bariátrica. Revista de Divulgação Científica Sena Aires, Valparaíso de Goiás, v. 1, n. 2, p. 163-170, jul./dez. 2012.

RAMOS, C. P.; MELLO, E. D. Manejo nutrológico no pós-operatório de cirurgia bariátrica. International Journal of Nutrology, Catanduva, v. 8, n. 2, p. 39-49, maio/ago., 2015.

RAVELLI, M. N. et al. Obesidade, cirurgia bariátrica e implicações nutricionais. Revista Brasileira em Promoção da Saúde, Fortaleza, v. 20, n. 4, p. 259-266, 2007.

ROCHA, J. C. G. Deficiência de Vitamina B12 no pós-operatório de cirurgia bariátrica. International Journal of Nutrology, Catanduva, v. 5, n. 2, p. 82-89, maio/ago. 2012.

SANTOS, M. T. A. et al. Alterações de parâmetros relacionados ao metabolismo ósseo em mulheres submetidas 
à derivação gástrica em Y de Roux. Arq Bras Endocrinol

Metab., São Paulo, v. 56, n. 6, p. 376-382, aug. 2012.

SCHAUER, P. R. et al. Bariatric surgery versus intensive medical therapy in obese patients with diabetes. N Engl J Med., Boston, v. 366, n. 17, p. 1567-1576, Apr. 2012.

SCHNEIDER, B. E.; MUN, E. C. Surgical management of morbid obesity. Diabetes Care, Alexandria, v. 28, n. 2, p. 475-480, fev. 2005.

SOCIEDADE BRASILEIRA DE CIRURGIA BARIÁTRICA E METABÓLICA. Consenso Brasileiro Multissocietário em cirurgia da obesidade. 2006. Disponível em: <http://www.sbcb.org.br/associados.asp?menu=2>. Acesso em: 12 jan. 2012.

STURM, R. Increases in morbid obesity in the USA: 2000-2005. Public Health, Amsterdam, v. 121, n. 7, p. 492-496, jul. 2007.

TAVARES, I. da S. et al. Função diastólica do ventrículo esquerdo em obesos graves em pré-operatório para cirurgia bariátrica. Arq. Bras. Cardiol., São Paulo, v. 98, n. 4, p. 300-306, abr. 2012.

TEICHMANN, L. et al.; Fatores de risco associados ao sobrepeso e a obesidade em mulheres de São Leopoldo, RS. Rev. Bras. Epidemiol., São Paulo, v. 9, n. 3, p. 360373 , set. 2006 .
TOPART, P. et al. Biliopancreatic diversion requires multiple vitamin and micronutrient adjustments within 2 years of surgery. Surgery for Obesity and Related Diseases, New York, v. 10, n. 5, p. 936- 941, Set./Oct. 2014.

TOREZAN, E. F. G. Revisão das principais deficiências de micronutrientes no pós-operatório do Bypass Gástrico em Y de Roux. International Journal of Nutrology, Catanduva, v. 6, n. 1, p. 37-42, jan./abr. 2013.

VALEZI, A. C. et al. A importância do anel de silicone na derivação gástrica em Y-de-ROUX para o tratamento da obesidade. Rev. Col. Bras. Cir., Rio de Janeiro, v. 35, n. 1, p. 18-22, fev. 2008.

WORLD HEALTH ORGANIZATION. Obesity and overweight. Geneva. 2009. Disponível em: <http://www. who.int/mediacentre/factsheets/fs311/en/index.html>. Acesso em: 30 out. 2017.

WORLD HEALTH ORGANIZATION. Obesity: preventing and managing the global epidemic. Report of a WHO consultation. Geneva: WHO, 2000.

YESTE, D.; CARRASCOSA, A. El manejo de la obesidad en la infancia y adolescencia: de la dieta a la cirugía. Endocrinol Nutr., Barcelona, v. 59, n. 7, p. 403-406, ago./ sep. 2012.

YOUSSEF, Y. Risk of secondary hyperparathyroidism after laparoscopic gastric bypass surgery in obese women. Surg Endosc., New York, v. 21, n. 8, p. 1393-1396, Aug. 2007. 